\title{
DETACHED MAIN-SEQUENCE BINARIES: A LABORATORY FOR THE STUDY OF STELLAR STRUCTURE
}

\author{
A. GIMENEZ \\ Laboratorio de Astrofísica Espacial y Física Fundamental (INTA) \\ Estación de Villafranca, Apartado 50727. 28080 Madrid, Spain.
}

\begin{abstract}
Our knowledge of the evolutionary processes taking place in interacting binary stars is based primarily in our understanding of the stellar structure and evolution of single stars. The determination of absolute dimensions in close doble-lined eclipsing binaries, with well-detached components, is the best empirical approach available into stellar structure.

Important physical parameters and processes can be studied in some detail within the framework of detached main-sequence binaries, e.g., initial chemical composition, the mixing-length ratio, the relevance of convective overshooting, interactions between matter and radiation in the stellar interior, the concentration of mass towards the center of the stars or the response of stellar envelopes to tidal influences by an external gravitational potential.

A combination of observational results from photometry and spectroscopy are used to obtain relevant stellar parameters: absolute dimensions, ages, chemical composition, apsidal motion rate, degree of synchronization and circularization, etc., and a comparison with theoretical models allow us to derive some information about the above mentioned physical mechanisms.
\end{abstract}

\section{Introduction}

Understanding stellar evolution is fundamental for building a coherent history of the Universe and the adoption of theoretical models of stellar evolution is at the basis of modern astrophysics research. Models are used to estimate average lifetimes at various evolutionary phases, to describe the transformation of chemical elements within the stars and the pollution of the interstellar environment with processed material or to predict stellar pulsation periods, but also to assign most probable stellar parameters, masses and radii, to single stars for which only limited photometric information is available.

Despite the large development in our knowledge of stellar structure and evolution since the beginning of stellar astrophysics, important problems are still present, even within the apparently simple phase of the main sequence. The solar neutrino flux or the spectrum of solar oscillations, stellar pulsation, energy generation rates, mass loss through stellar winds, opacity of stellar matter or the convective transport of encrgy, are just some examples of research areas needing further theoretical insight, and as much observational information as possible.

Current stellar evolution theories characterize a star by its mass, initial fraction of helium and metals, and age, for a given mixing-length parameter, degree of convective overshooting, opacity library, nuclear reaction rates and equation of state. Three kinds of astrophysical object permit us to test the adopted theoretical models and their input physics: the Sun, eclipsing binaries and star 
clusters. With the information derived from these sources, we intend to test how well do theoretical computations reproduce real stars for various levels of sophistication in the adopted input physics and to quantify as many as possible of the free parameters in the models. This is obviously possible only when we have more observed parameters (at least 4) than needed to establish the corresponding theoretical model to compare with. For the Sun, we have observational data of the mass, radius, temperature, age and metal abundance. In the case of open clusters, a common age and chemical composition for all member stars is generally assumed, and individual masses and radii can be accurately measured for the component stars of double lined eclipsing binaries. Additional sources of information, like the flux of neutrinos or the acustic oscillations spectrum in the Sun, or the apsidal motion rates in eclipsing binaries, can be used to test a number of adopted parameters in the theoretical models. In fact, current standard models of stellar evolution are suspect because they predict a solar neutrino flux about a factor of 3 larger than the observed value and, according to the measured apsidal motion rates of some well-detached binaries, real stars seem to be significantly more centrally condensed than predicted by theory.

Measuring the acustic frequencies of solar oscillations, we have access to check the speed of sound in the solar interior and recent results have shown the location of the base of the solar convective envelope or rotational rates below the photosphere (see, e.g. Christensen-Dalsgaard, 1988). The mixing-length parameter, $l / H_{P}$, for which no theoretical estimate is available, is fixed from the observed solar dimensions so that the radius, metal abundance and age fit the adopted model, but the mixing-length theory of convection is only a crude approach to the real situation and we should test the validity of a single value for all masses and ages. On the other hand, the important model parameter of the initial fraction of helium, Y, which is not easy to measure, is also generally assumed for all systems in our neighborhood from solar data.

The study of open clusters has provided important additional information about stellar structure, particularly, the location of the TAMS in the HR diagram. Maeder and Mermilliod (1981) have found an extension of 0.6 to 0.8 magnitudes in the width of the main sequence with respect to predictions based on standard models that could be explained in a natural way if the convective cores of the stars present a moderate amount of overshooting of about 0.25 in terms of the pressure scale height. These results appeared nevertheless to be very controversial since different studies suggested either larger values (e.g., Mazzei and Pigatto, 1988) or much smaller, even no overshooting at all (e.g., Stothers and Chin, 1991). A comparison of the obtained cluster diagrams with theoretical isochrones demands though a careful test of membership and binarity of the candidate stars (Nordström and Andersen, 1991).

But, the most fundamental property of a star is obviously its mass, and the only direct way of measuring it is from the study of double-lined eclipsing binaries. Detached main-sequence binary stars are thus the main laboratory for the study of stellar structure. Besides this, the reason for the inclusion of this paper in the present meeting is, in fact, that they also provide scenarios for the formation of the more exotic and interacting stars. A correct understanding of the physics in the earlier, detached, phases of binary systems leading to mass transfer episodes is certainly needed to be able to attempt modelling later phases and the involved evolutionary processes. Some procedures and results are discussed here but, for a more detailed description of the present status in the determination of accurate masses and radii of normal stars, we refer to Andersen (1991)

\section{How to obtain accurate data}

In order to test the subtle effects we are looking for in the models, measuring stellar dimensions by observing double-lined eclipsing binaries is only fruitful if the observational precision is about 
$\pm 1 \%$ for the masses, $\pm 2 \%$ for the radii and $\pm 10 \%$ for the luminosities of both components, assumed to have the same age and initial chemical composition. Methods to reach this precision have been already discussed by Andersen et al. (1980).

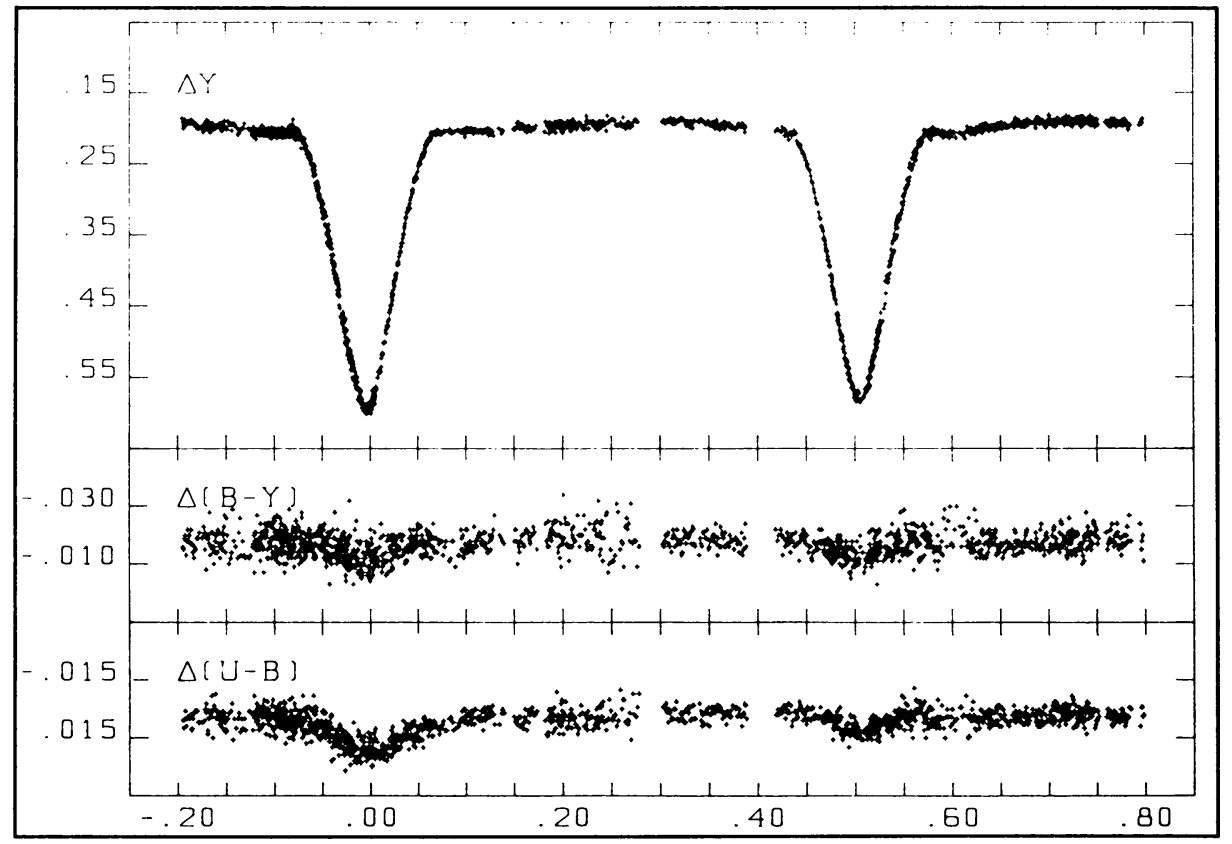

The kind of binaries needed for these studies are trivial systems, non-interacting stars with welldetached components. The observations required are both photometric and spectroscopic. Photometry provides multicolour light curves, colour indices and times of minima. Spectroscopic studies provide radial velocity curves and metal abundance determinations. A combination of all sources of information allow an accurate determination of masses and radii, effective temperatures, luminosities, chemical composition and the average internal structure constant. In order to obtain high-precision results though, the analysis is far from trivial, even if the binary system and the observational material have been adequately selected. Spectroscopic elements present difficulties in the obtention of the radial velocity curve due to well-known problems with the selection of reliable unblended lines though, once a good radial velocity curve is obtained, the corresponding orbital elements are rather straightforward to derive. In the case of the photometric parameters, it is nowadays not very difficult to obtain a good multicolour light curve but, in order to derive consistent elements out of it, a careful analysis with a realistic synthetic modelling code should be made. Andersen et al. (1980) have shown examples of the two problems: systematic errors in the determination of the masses by the use of inadequate spectral features and the equally good representation of a high-quality light curve (QX Car) with two quite different solutions. In Figure 1 we show the light curve of the slightly eccentric early-type binary CW Cep (Clausen and Giménez, 1991), with a V magnitude of 7.8 and an orbital period of 2.7 days, in the Strömgren $y$ filter, as an example of the data used in this kind of studies.

Usual problems to obtain a good sample of reliable absolute dimensions are: selection effects, which prevent us from the possibility to observe any combination of component stars, the 
probability to obtain a clean light curve which decrease for low-mass or evolved stars due to activity-related phenomena, line broadening and overlapping which is linked to short orbital periods, and theoretical modelling of proximity effects. Due to these problems, there are several weak areas in the $\log g$ - $\log T$ diagram that deserve further observational attention. Low-mass stars, below that of the Sun, or very hot stars, of more than 15 solar masses, with accurate absolute dimensions are still scarce. On the other hand, binaries close to the ZAMS, with $\log g_{\text {ZAMS }}-\log g_{\text {obs }}$ $<0.02$, and evolved systems beyond the TAMS are also lacking. Of course, further examples in different environments than that of the solar neighborhood would be most rewarding, like binaries in the halo population or in external galaxies.

The case of massive stars is particularly illustrative. Important changes in the input physics and the internal structure of these stars have been reported that need to be tested before their generalized application to more evolved phases. Unfortunately, few massive stars have good absolute dimensions. Reasons are known to be the small amount of available undisturbed systems, the relatively few strong lines in the optical spectrum, that large stars and short periods imply large rotational velocities leading to broad and shallow lines, and the difficulties arising from temperature calibrations. Popper and Hill (1991) recently warned against overoptimistic determinations of stellar masses for hot binaries and proposed the use of calibrations by synthetic spectroscopic systems to test for systematic errors in the use of cross-correlation techniques.

Compilation of absolute dimensions derived from close binaries have been published by several authors and, most recently by Harmanec (1988) and Andersen (1991). Fundamental stellar properties are generally collected from the literature in order to provide average relations in terms of spectral type or effective temperatures valid for single stars. Global analyses of this kind are nevertheless not justified if evolutionary parameters and initial chemical composition differences are not adequetely taken into account. Nordström (1989) already prevented against the use of a unique spectral type - mass relation suggested by previous authors. The validity of such a relation, even for main-sequence stars, carries an inherent uncertainty of some $\pm 5-15 \%$ error due to the effects of evolution within the main-sequence band for an uncertainty of \pm 1 subtype. In Figure 2 , we have plotted a mass-radius relation for the individual components of binary systems using data by Popper (1980). The scatter shown is not due to observational errors, and thus information is lost when one-dimensional formulae are used. For a given temperature, there is a width of \pm 0.2 in $\log \mathrm{R}$ and, in terms of spectral type, stellar radii may vary more than $50 \%$. A two-parameter approach, or even three (to take into account not only the effect of evolution but also that of metal abundances), has been proposed by Andersen (1991) to allow a realistic prediction of fundamental dimensions in single stars using data derived from the study of binary systems. Calibrations of this type are nowadays possible thanks to the high precision of the available absolute dimensions but we should not forget the existing weak regions in the HR diagram, as commented above, which may compromise the validity of the approximation formulae.

\section{The test of theoretical models}

The main input physics elements to be tested using binary stars are: radiative opacities, the mixing-length parameter, convective-core overshooting and stellar wind mass loss. Nevertheless, we will concentrate only in the results obtained up to now concerning opacities and overshooting. The mixing length ratio is currently adopted from solar data since few low-mass binaries with accurate dimensions are still available and independent metal abundance determinations are needed before any serious attempt is made to derive new values. The applicability of the same value for stellar configurations of different mass and age is anyhow far from clear. On the other hand, 


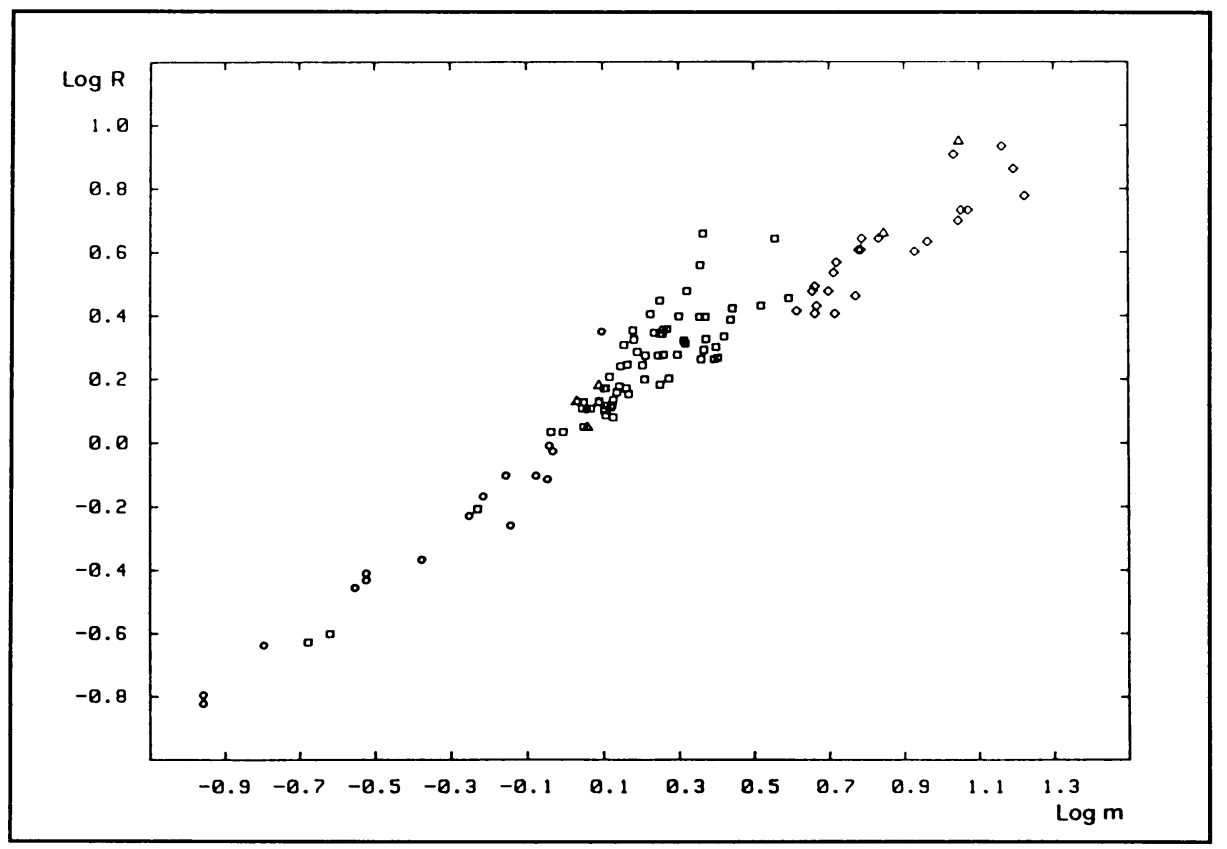

stellar wind mass loss is mainly effective in the more massive range of stars and these are known to show several problems in the determination of accurate dimensions, as commented above, that difficult the obtention of reliable results.

Data derived from the study of binary stars are not only used for testing the quality of theoretical models but also to quantify the relevance of the various physical mechanisms included. Due to our poor knowledge of the above mentioned input physics elements, the comparison between observations and models is rather interactive and binary stars are used to derive several parameters which are later to be adopted in other areas of astrophysics. Calibration of these parametric approaches, e.g. to convection, can not be made with the Sun which represents a single point in the HR diagram. The analysis of double-lined eclipsing binaries to test theoretical models of stellar structure is indeed an old method which has been recently renewed by improved accuracies. A 1-2\% accuracy in masses and radii gives very good resolution in the main sequence and pinpoints a star of fixed mass within $1 / 20$ of the width of the main sequence. Binaries with mass errors of just $5 \%$ do not provide any new information on current main-sequence models.

The treatment of energy transportation in the interior of the stars is in fact one of the weaker points of our knowledge of stellar structure. The currently adopted opacity tables from Los Alamos, compared to Cox \& Stewart opacities, lead to cooler and less luminous stellar models specially significant in the low-mass range. In the case of convective transport, the classical mixing-length approximation when corrected through parametric overshooting into the, classically stable, radiative regions produce brighter and longer-lived models. Computations of evolutionary models for core-hydrogen burning phases, with special attention to the effects of different convection theories, have been revitalized by the good quality of the now available observational data (Claret and Giménez, 1991a). Most proposed tests of overshooting concern evolved stars (Maeder and Meynet, 1989; Stothers, 1991) for which stellar mass is basically unknown. 
Extremely conservative limits to convective overshooting $\left(d / H_{P}<1.7\right)$ have been derived from the location of the ZAMS defined by the component stars of binary systems by Stothers and Chin (1990). The small sensitivity of ZAMS models to the amount of overshooting and the few really unevolved binaries with accurate dimensions available, make the use of binaries located around the TAMS more suitable for this kind of studies.

Binaries in the mass range between 1.5 and 2.5 solar masses at the top of the main sequence were found to require some amount of overshooting to explain their position in the HR diagram with a common isochrone for both components of the same system, as it was shown by Andersen et al. (1990). On the other hand, binaries with lower mass, like AI Phe (about 1.2 solar masses) and UX Men, were found to show a good fit to theoretical models without overshooting when an appropriate opacity library was used (Andersen et al., 1988 and 1989). Should this imply that there is a change with stellar mass in the need of convective overshooting to explain the observations? Clausen (1991) did analyze the case of BW Aqr, an F-type binary at the top of the main sequence with masses around 1.45 in the transition region where stars develop convective cores and found that a good fit for a common age ( 2.5 Gyears) was only possible with a moderate amount of overshooting. The same was suggested by the somehow similar system BK Peg using data by Popper (1983). On the other hand, overshooting was not found to be necessary to understand the evolutionary status of the RS CVn-type binary V792 Her studied by Fekel (1991) with masses in the same range. A detailed study of a larger sample of these active binaries is nowadays possible thanks to the effort by Popper $(1988 \mathrm{a}, 1988 \mathrm{~b}, 1990)$ to obtain precise radial velocity curves of this type of systems. A preliminary analysis of the available information suggest that in fact overshooting seems to be unnecessary to explain them despite the systematic location of the primary components close to the top of the main sequence. This is probably the only plausible position to reconcile their age with the position of the secondaries, with very similar mass, at the bottom of the giant branch. In the massive range, nothing can be said yet because only relatively unevolved binaries, like EM Car, have good absolute dimensions and a moderate amount of overshooting does not make any significant difference except for the internal density concentration. Analyses of Wolf-Rayet/O Star binaries indicate nevertheless that overshooting is a small effect in massive stars (Wheeler, priv. comm.). It should be emphasized that the information provided by binary systems is not distance dependent and that the location of the TAMS is not the only constraint given by the comparison with theoretical models but the fit to a common isochrone of both components. Revisions of the TAMS through changes in the metal abundances of the models (Stothers, 1991) are therefore not sufficient to explain the observations.

Additional quantities that can be measured accurately in some eclipsing binaries are the apsidal motion constant which measures the average internal density concentration of the component stars, the level of synchronization of their rotational velocities with the orbital angular rotation or the circularization of the orbit. Comments about recent results of studies in these important areas for observational testing of stellar structure are given in this volume by Giménez and Claret (1991) and Claret and Giménez (1991c). Apsidal motion measurements seem to favour the existence of some amount of overshooting (Claret and Giménez, 1991b), but only after definitive parameters are obtained for the more evolved systems will a quantitative evaluation be possible.

Concerning the initial chemical composition of binary stars, we have seen that significant changes in $\mathrm{Z}$ are expected from system to system. As commented above, a reliable empirical ZAMS is not yet defined by the too few binaries at hand and this region is very important due to its sensitivity to the adopted metal content in the models. A change of \pm 0.01 in $\mathrm{Z}$ shifts the ZAMS \pm 0.05 in $\log \mathrm{g}$. In the low-mass range, the position of the ZAMS is also very dependent on the adopted opacities. For normal binaries though a single value of $\mathrm{Z}$ is not valid and metal 
abundances have to be determined independently through high-dispersion spectroscopic observations leading to well-defined absorption profiles of selected metallic lines.

On the other hand, helium is the second most abundant element in the interstellar medium but unfortunately is very difficult to determine its contribution to the chemical composition of real stars, even for the Sun. The value of $\mathrm{Y}$ is a basic parameter to fit theoretical stellar evolution tracks to cluster colour-magnitude diagrams. Popper and Ulrich (1986) studied the binary in the Hyades HD 27130 and concluded that a solar helium abundance was the best choice to reproduce the observed physical parameters. A global study of available binaries with accurate masses, radii and temperatures, provides indications that a good fitting is possible with an adopted value of $Y$ $=0.28 \pm 0.02$, though metal abundances may be significantly different for each individual case. The same result was also obtained by Andersen et al. (1988) with the well-detached binaries AI Phe and UX Men. These later authors also showed that the previously reported anomalously low values obtained for late-type stars (Andersen et al., 1984) could be explained in terms of the inadequate opacities adopted. A revision of Hejlesen (1980) theoretical models by Claret and Gimenez (1989) with Los Alamos opacity library clearly demonstrated the mentioned effect.

One should keep in mind that, again, a new set of opacity tables with improved physics, mainly concerning the equation of state, the efficiency of metals in the interactions between radiation and matter, and the presence of molecules, by Iglesias and Rogers (1991) have been computed which appear to supersede those from Los Alamos. The effect in the interpretation of absolute parameters from binary systems should be carefully tested but they seem to improve the comparison with observations concerning the depth of the convective envelope in the Sun and models for late-type stars are expected to be systematically cooler and with larger radius than those computed with the previous opacity library. A similar behaviour to that found when changing from Cox-Stewart to Los Alamos in the $80^{\prime}$ is now foreseen for the $90^{\prime}$.

\section{Prospects for future research}

Given the most recent improvements in the input physics of stellar structure models, a new grid of models should be made available for various metal contents and degrees of overshooting. The results of computations by two independent groups at Lawrence Livermore (OPAL) and the international group of the Opacity Project (OP) seem to show a rather good agreement despite the complexity of the physics and computing procedures involved. Improved opacities as those obtained by Iglesias and Rogers (1991) may in fact decrease the need for convective overshooting. On the other hand, convective transport of energy may also receive new ideas and a good example is the new model of stellar turbulent convection, introduced by Canuto and Mazzitelli (1991), which could replace the current mixing-length theory. Preliminary results seem to agree with observed values for the Sun but a throughful check has yet to be done. It is interesting to note that overshooting is included in this theory free of adjustable parameters.

Detached binaries that deserve more observational and theoretical attention are abundant. Concerning the coverage of the HR diagram with good stellar dimensions, massive hot binaries like EM Car and beyond are badly needed, despite the known problems of temperature calibration, as well as systems with masses below that of the Sun. From the point of view of the different evolutionary stages, evolved binaries beyond the TAMS (normal red giants similar to TZ For anlyzed by Andersen et al., 1991) are known to be very rewarding though difficult to study, and pre-main sequence systems are also scarce and difficult due to circumstellar material and activity features. The case of EK Cep, shown by Popper (1987) to have a pre-main-sequence secondary component, is a good example of clean system, probably due to the blow away of material by the 
hot companion.

New steps in the near future should also be directed in the determination of accurate absolute dimensions of the component stars in close binaries of increasing level of interaction. The obvious first step is the analysis of RS CVn-type binaries, still detached but with the more evolved component close to its critical surface and evidences of having suffered some kind of enhanced stellar wind mass transfer. The second approximation to more exotic interacting binaries within the realm of high-precision stellar astrophysics would be the study of Algol-type binaries. Popper $(1988 \mathrm{a}, 1988 \mathrm{~b}, 1989,1990)$ has already advanced in this directions by producing good radial velocity curves for the two types of binary systems.

The membership of an eclipsing binary in a star cluster or association can provide important additional tests of the stellar evolution theory. The careful analysis of well-defined light and radial velocity curves yield the absolute dimensions and the masses of the components and these provide independent determination of age, chemical composition, and distance for the binary and the cluster in which it resides. A first example of this kind of studies, already commented, was the case of the binary in the Hyades by Popper and Ulrich (1986) showing that a standard solar chemical composition was clearly adequate for this classical and controversial cluster. Further examples, e.g. by Giménez et al. (1986), Clausen and Giménez (1991) or the systematic research by Milone and collaborators (some examples are shown in this volume) have certainly reinforced our confidence in this area of research and catalogues of eclipsing binaries in the fields of open clusters have become available (Clausen and Gimenez, 1987; Popova and Kraicheva, 1984) to help in the selection of stars for future studies.

One important advance in recent observational techniques is the use of CCD detectors for stellar photometry. This makes possible nowadays to study faint binaries of special interest in our Galaxy, like the lower end of the main sequence, as well as in other nearby galaxies. The presence of eclipsing binaries in the Large and Small Magellanic Clouds, as well as in M31, has been known for several decades. Unfortunately, the low precision of photographic light curves and the complete lack of radial velocities prevented from the obtention of relevant scientific results. The use of more efficient detectors such as CCD's, make possible high-precision photometry of eclipsing binaries in the LMC and SMC with moderate size telescopes as shown by Jensen et al. (1988) who obtained light curves with an average precision of \pm 0.01 magnitudes. As an example, the light curve obtained for the system HV12634 with an orbital period of 1.19 days and V magnitude of 15.2 is shown in Figure 3, which can be compared with that in Figure 1 for a 7.4 magnitudes brighter galactic binary. Unfortunately, few direct measures of the masses of the component stars in binary systems of another galaxy have been reported. Only some cases have been investigated by Niemela (1987, and this volume) and Bell et al. (1991). An interesting case to be further studied is the binary system HV2274 in the LMC described by Watson et al. (1991), very probably detached and showing a slightly eccentric orbit. As discussed by Koch (1990), the study of eclipsing binaries in other galaxies is important for several reasons: it should be possible to establish empirical mass-luminosity relations for stars with vastly different evolutionary and chemical history, it provides a chance to study the structure and evolution of stars in environments that may be systematically different from the solar neighborhood, and binaries with welldetermined physical properties may be used as accurate distance indicators to other galaxies (de Vaucouleurs, 1978) leading to an improvement in the extragalactic distance scale.

The most modern version of these new detectors will be implemented in the space project PRISMA, now under phase-A study within the European Space Agency. The possibility to obtain high-precision photometry, of around $10^{-6}$ magnitudes, opens the field of asteroseismology also to well-detached binaries providing periods of oscillation whose interpretation will contain new 


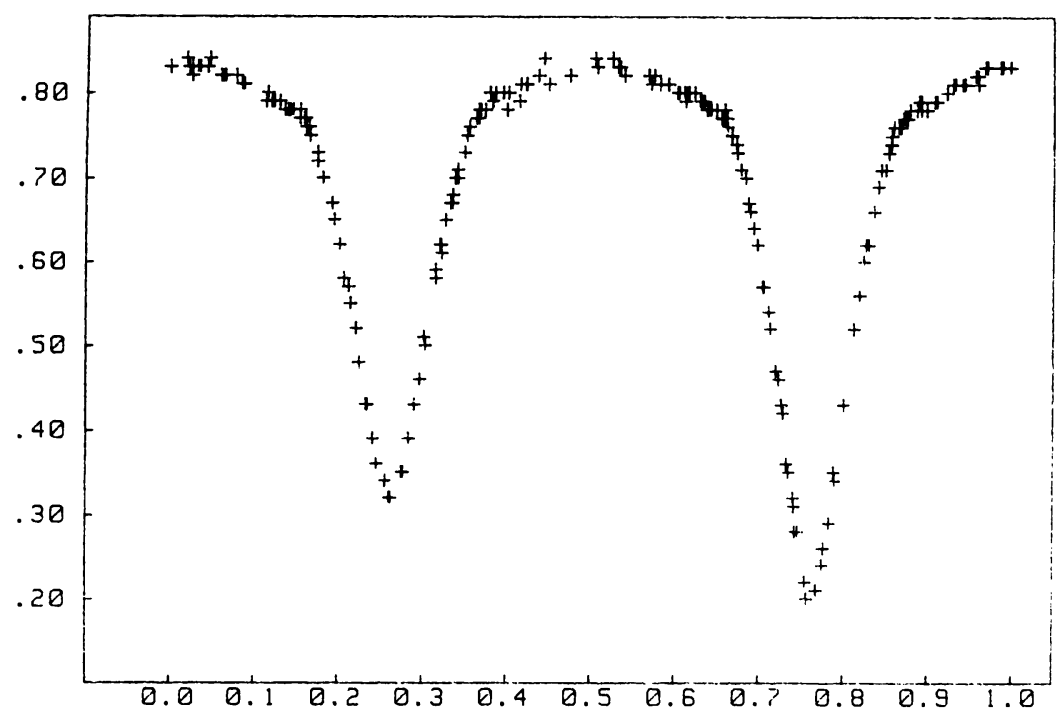

important hints about the internal structure of normal stars. On the other hand, the accurate distance determinations which are expected to result from the observing program of HIPPARCOS will also add, no doubt, new information for understanding interacting and detached binary systems, though only a small sample of nearby candidates are included.

Acknowledgements. Many inputs have been provided by Dr. J. Andersen but I would like to thank also Drs. J.V CLausen and A. Claret for innumerable comments and discussions. Support by the Spanish DGICYT has been provided through project PB87-0235.

\section{References}

Andersen, J. (1991), Astron. Astrophys. Rev., preprint.

Andersen, J., Clausen, J.V., Gustafsson, B., Nordström, B., VandenBerg, D.A. (1988), Astron. Astrophys., 196, 128.

Andersen, J., Clausen, J.V., Jorgensen, H.E., Nordström, B. (1984), in Observational Tests of the Stellar Evolution Theory (IAU Symp. No. 105), eds. A. Maeder \& A. Renzini. Reidel, Dordrecht, p. 397.

Andersen, J., Clausen, J.V., Magain, P. (1989), Astron. Astrophys., 211, 346.

Andersen, J., Clausen, J.V., Nordström, B. (1980), in Close Binary Stars: Observations and Interpretation (IAU Symp. No. 88), eds. M.J. Plavec, D.M. Popper \& R.K. Ulrich. Reidel, Dordrecht, p. 81.

Andersen, J., Clausen, J.V., Nordström, B., Tomkin, J., Mayor, M. (1991), Astron. Astrophys., 246, 99.

Andersen, J., Nordström, B., Clausen, J.V. (1990), Astrophys. J., 363, L33.

Bell, S.A., Hill, G., Hilditch, R.W., Clausen, J.V., Reynolds, A.P., Giménez, A. (1991), Mon. Not.

R. Astron. Soc., 250, 119. 
Canuto, V.M., Mazzitelli, I. (1991), Astrophys. J., 370, 295.

Christensen-Dalsgaard, J. (1988), in Seismology of the Sun \& Sun-like Stars, ESA Publication SP-286, p. 431.

Claret, A., Gimenez, A. (1989), Astron. Astrophys. Suppl., 81, 1.

Claret, A., Giménez, A. (1991a), Astron. Astrophys. Suppl., 87, 507.

Claret, A., Giménez, A. (1991b), Astron. Astrophys., 244, 319.

Claret, A., Gimenez, A. (1991c), this volume.

Clausen, J.V. (1991), Astron. Astrophys., 246, 397.

Clausen, J.V., Giménez, A. (1987), Proceedings of the 10th Europ. Reg. Astron. Meeting of the IAU, ed. J. Palous. Praha, vol. 4, p. 185

Clausen, J.V., Giménez, A. (1991), Astron. Astrophys., 241, 98.

De Vaucouleurs, G. (1978), Astrophys. J., 223, 730.

Fekel, F.C. (1991), Astron. J., 101, 1489.

Giménez, A., Claret, A. (1991), this volume.

Giménez, A., Clausen, J.V., Andersen, J. (1986), Astron. Astrophys., 160, 310.

Harmanec, P. (1988), Bull. Astron. Inst. Czechosl., 39, 329.

Hejlesen, P.M. (1980), Astron. Astrophys. Suppl., 39, 347.

Iglesias, C.A., Rogers, F.J. (1991), Astrophys. J., 371, 408.

Jensen, K.S., Clausen, J.V., Giménez, A. (1988), Astron. Astrophys. Suppl., 74, 331.

Koch, R.H. (1990), in Active Close Binaries, ed. C. Ibanoglu. Kluwer, Dordrecht.

Maeder, A., Mermilliod, J.C. (1981), Astron. Astrophys., 93, 136.

Maeder, A., Meynet, G. (1989), Astron. Astrophys., 210, 155.

Mazzei, P., Pigatto, L. (1988), Astron. Astrophys., 193, 148.

Niemela, V. (1987), in Luminous Stars and Associations in Galaxies (IAU Symp. No. 116), eds.

C.W.H. de Loore, A.J. Willis \& P. Laskarides. Reidel, Dordrecht, p. 85.

Nordström, B. (1989), Astrophys. J., 341, 934.

Nordström, B., Andersen, J. (1991), ESO Messenger, 63, 34.

Popova, M., Kraicheva, Z. (1984), Astrofiz. Issled. Izv. Spets. Astrofiz. Obs., 18, 64.

Popper, D.M. (1980), Ann. Rev. Astron. Astrophys., 18, 115.

Popper, D.M. (1983), Astron. J., 88, 1242.

Popper, D.M. (1987), Astrophys. J., 313, L81.

Popper, D.M. (1988a), Astron. J., 95, 1242.

Popper, D.M. (1988b), Astron. J., 96, 1040.

Popper, D.M. (1989), Astrophys. J. Suppl., 71, 595.

Popper, D.M. (1990), Astron. J., 100, 247.

Popper, D.M., Hill, G. (1991), Astron. J., 101, 600.

Popper, D.M., Ulrich, R.K. (1986), Astrophys. J., 307, L61.

Stothers, R. (1991), Astrophys. J., in press.

Stothers, R., Chin, Ch.-w. (1990), Astrophys. J., 348, L21.

Stothers, R., Chin, Ch.-w. (1991), Astrophys. J., in press.

Watson, R.D., West, S.R.D., Tobin, W., Gilmore, A.C. (1991), this volume. 